\title{
Synaesthetic Audio-Visual Sound Toys in Virtual Reality
}

\author{
Jonathan Weinel \\ School of Mathematical and \\ Computer Sciences \\ University of Greenwich \\ J.R.Weinel@greenwich.ac.uk
}

\begin{abstract}
This paper discusses the design of audio-visual sound toys in Cyberdream, a virtual reality music visualization. While an earlier version of this project for Oculus GearVR provided a journey through audio-visual environments related to 1990s rave culture, the most recent iteration for Oculus Quest provides the addition of three audio-visual sound toys, the discussion of which is the main focus of this paper. In the latest version, the user flies through synaesthetic environments, while using the interactive controllers to manipulate the audio-visual sound toys and 'paint with sound'. These toys allow the user to playfully manipulate sound and image in a way that is complementary to, and interfaces with, the audio-visual backdrop provided by the VR music visualization. Through the discussion of novel approaches to design, the project informs new strategies in the field of VR music visualizations.
\end{abstract}

\section{CCS CONCEPTS}

- Computing methodologies $\rightarrow$ Computer graphics $\rightarrow$ Graphics systems and interfaces $\rightarrow$ Virtual reality

\section{KEYWORDS}

Music visualizations, Virtual reality, VJ, Audio-visual performance, Sound design.

\section{Introduction}

Audio-visual performance is a well-established field, which encompasses a range of creative practices. Within this area, virtual reality (VR) music visualization is a growing field [7], which includes many interesting opportunities for new forms of audio-visual composition and experience to emerge. In Rajmil Fischman's discussion of 'music in the holodeck' [4], he calls for accessible, interactive experiences that allow participants to create electronic sound in immersive environments. Drawing upon the idea of the holodeck (a fictional immersive multisensory environment depicted in the TV show Star Trek), Fischman proposes the use of gestural technologies such as the data glove, which allow participants to interactively shape and manipulate sound intuitively, thereby making the composition of electroacoustic music accessible to non-experts as a creative social experience. Recent forms of VR could provide good platforms for designing experiences of the type Fischman points towards. Yet, while there is existing work exploring the design of sound toys in VR [6], the visual component of these experiences is typically used to provide graphical user interfaces and/or representations based on the appearance of real-world musical instruments. While these are valid approaches, in VR we do not necessarily need to design instruments that imitate those already available in the physical world. By drawing on existing practices in visual music and VJ performance, we can conceive of audio-visual sound toys that generate sounds and corresponding abstract imagery.

Contributing towards research in this area, Cyberdream is a project that emerged as a progression of my earlier work in audiovisual composition [8]. This work draws upon the approaches of VJing [3], in which electronic dance music tracks are combined with complementary visuals, taking the audience on an audiovisual journey. Cyberdream extends this idea, translating these approaches into the domain of VR, in order to provide immersive spatial visualizations of music. While my VJ mixes provide visual montages that are projected in front of an audience, Cyberdream adapts these aesthetic forms for VR, placing the user inside 3D scenes related to music. Originally this piece was created for the Oculus GearVR, an untethered VR headset, providing a journey through synaesthetic audio-visual environments that is conceptually based on 1990s rave culture [9]. The project was later rebuilt and expanded for the Oculus Quest headset, which provides an improved quality of experience, leading to the current version of Cyberdream that this paper discusses.

Cyberdream draws upon the aesthetic approaches of breakbeat hardcore music. This genre reflects a set of aesthetic priorities that find their historic origins in early 1990s UK rave music, however, it can also be understood as a genre which remains active, as various producers continue to explore this style for the benefit of contemporary audiences. Record labels such as Kniteforce, Sneaker Social Club, Lost City Archives, and Of Paradise, are among those currently releasing breakbeat hardcore music. While breakbeat hardcore has a sonic style, it also has a visual language that was established in the 1990s through the graphical art found on cultural artefacts such as record sleeves and event flyers [1]. 
By coupling breakbeat hardcore music with related visuals, Cyberdream creates an audio-visual experience which is comprehensible for contemporary audiences of rave culture. However, it also provides something entirely new, since this music and imagery has never been experienced together like this in the form of an interactive VR experience. By situating the user in a 3D audio-visual spatial experience of music, the project forges new approaches in audio-visual composition and breakbeat hardcore that are specific to VR. Furthermore, in the most recent version of this project for Oculus Quest, three audio-visual sound toys also allow the user to generate sounds that form part of the experience. Following an explanation of the conceptual and structural approaches used by Cyberdream, these three toys will be discussed in more detail.

\section{Synaesthetic Music Visualizations}

The approach taken for visualizing music in Cyberdream is one of 'symbolic representation', whereby associative features of the music genre being visualized are derived and used as a basis for visual design [10]. In simple terms, when using this method, surf rock music might be visualized using symbolic images of surfing, while heavy metal music might be visualized as skulls in pits of fire. In either case, these are images that are readily associated with the iconography of the respective music genres, as established through recurring visual themes found on album covers, event flyers, and other cultural artefacts. This approach can also be observed in some examples of VJing or live visuals projected at concerts. For instance, gabber events such as Masters of Hardcore [5] are based around a specific style of electronic dance music that conveys 'dark' or violent themes, via the use of distorted synthesizers and sampled material gathered from horror films and gangster rap music. The recurring visual style of gabber transmits these themes via images of skulls, horror masks, weapons, or demonic characters. These can be found on the record sleeve artwork, websites, clothing, event flyers, and video projections at gabber events. If we were to look at other styles of music, we would similarly find visual languages present in various cultural artefacts, and these languages would be interwoven with themes present in the music, which are communicated through aspects of lyrics, sound design, and music production. By noticing these symbolic languages, we can use them as a basis for the design of music visualizations, while also contributing towards their evolution, and gradually redefining them.

This symbolic approach to music visualization can also be used in combination with other techniques. For example, a symbolic visualization could also capture properties of an audio signal such as frequency or amplitude, and use these to generate patterns or modify visual filters. However, it is important to emphasize that the main objective of this symbolic approach is to access a deeper conceptual visual language, which relates to cultural constructs embedded in the music. By accessing this visual language, it can be harnessed as a basis for the generation of images.

This approach to music visualization can also be understood as 'synaesthetic'. Synaesthesia describes a blurring of the sensory modalities, whereby sounds may have a color, a smell, or a taste, for example [2]. A person having a synaesthetic experience of music, whilst listening on headphones might therefore perceive visual impressions that arise as a manifestation of the communicative properties of sound. Music visualizations can imitate this process, by taking associative properties of sound and using them to generate concrete synthetic visuals, which audiences can then watch on digital displays. In this regard, we might reasonably describe these music visualizations as having synaesthetic qualities.

\section{Structural Design}

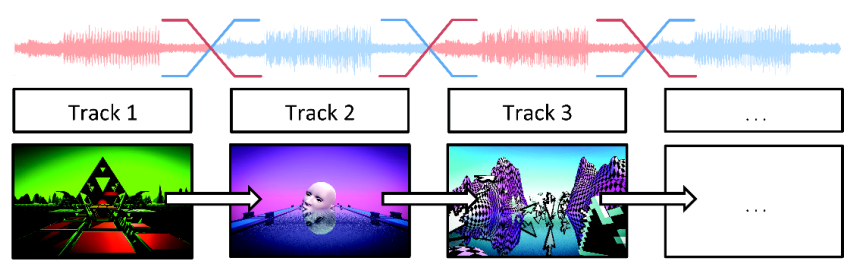

Figure 1: The structure of Cyberdream creates an audiovisual backdrop analogous to a DJ or VJ mix.

Cyberdream is a synaesthetic music visualization that utilizes the symbolic approach described, providing a series of $3 \mathrm{D}$ environments based on the typical imagery seen on 1990s rave fliers, which depict futuristic and surrealistic themes [1]. The project was created in the game engine Unity, by making a series of original scenes using the in-built terrain tools in Unity, which arrange various 3D models, textures, and skyboxes. Scripts were written in $\mathrm{C} \#$ in order to provide interactive features and animations such as oscillating visual effects. During the experience, the user flies through these scenes on an automated path.

Each 3D scene has a corresponding electronic dance music track, composed specifically for this project in a breakbeat hardcore style. These compositions were made in the music tracker Renoise, using breakbeat samples and virtual instruments from the Roland Cloud suite, which recreates many of the synthesizers and drum machines which were used in 1990s electronic music. In combination with the 3D scenes, the music forms an audio-visual backdrop equivalent to a DJ or VJ mix, which the user flies through in VR. Figure 1 illustrates this structural approach. At the end of each scene, a fading transition occurs which brings the next one in visually, while the music cross-fades in synchronization, just as it would do in a DJ mix. The latter effect is technically accomplished using game audio middleware tool Wwise, by configuring the various tracks using timing information and transition points.

While the progression through 3D scenes provides the macro structural form of the piece, the addition of three audio-visual sound toys also allows the user to interactively create sounds and images that provide complementary, micro structural forms. 


\section{Audio-Visual Sound Toys}

\subsection{Zig-Zag Toy}

Zig-Zag Toy emits jagged orange beams and percussive pulsing sounds (Figure 2). The source materials for this toy are percussive sounds that were made using a Korg MS-20 analogue synthesizer. These sounds are appropriate to the breakbeat hardcore style, and are designed as a complementary layer of percussive material that could fit with any of the tracks. Using the Oculus Touch controller, the user points in a direction and pulls the primary trigger to activate the beam. When active, the beam generates a series of points at equal distances along its length, which randomly offset the coordinates, producing a jagged pattern.

The beam is synchronized with the electronic dance music track heard in the given scene and emits sequences of pulsing sounds. These pulses are stored in binary sequences using bit flags (Figure 3 ), where a ' 1 ' signals that a beat is on, and a ' 0 ' signals that it is off. This allows looping rhythmic patterns to be stored, in a similar manner as one would find on a drum machine. Synchronization with the electronic dance music track is accomplished using callbacks from the Wwise audio engine, which provides timing information about the music. The system used compensates for latency by triggering pulses preemptively.

Using the secondary trigger on the controller also allows the user to switch between different patterns. The user may use any of the three sound toys in either hand, and if using two instances of the Zig-Zag Toy, it is possible to manipulate the primary and secondary triggers in order to create different interlocking patterns.

Additionally, the sounds of the right trigger are slightly offset by a few milliseconds, so that simultaneous pulses create an effect similar to what is known as a 'flam' in drumming, whereby a grace note is sounded before the main stroke, in order to thicken the sound. Using this approach, pulses trigged on the beat at the same time with the left and right controllers are distinct and produce a satisfying thickening of the sound. In addition, the left and right sounds are also offset spatially, giving a stereo effect.

Each time a pulse is emitted, the randomized locations on the beam update, changing their coordinates, so that with each pulse, the beam twists in time with the music. The sounds of the pulses can also be transformed by using the joystick (thumbstick) on the controller. Moving the stick up, down, left, or right, with the $x / y$ axes, activates digital signal processing (DSP) filter and pitch-shift effects, providing modifications to the sounds, such as one might encounter in a breakbeat hardcore track. In correspondence with these changes, the visual appearance of the beam is also modified, using oscillating effects, which shift the color values. In this way, Zig-Zag Toy allows the user to trigger sounds and images that can be simultaneously transformed, and which are designed to be complementary to the audio-visual backdrop of the given scene.

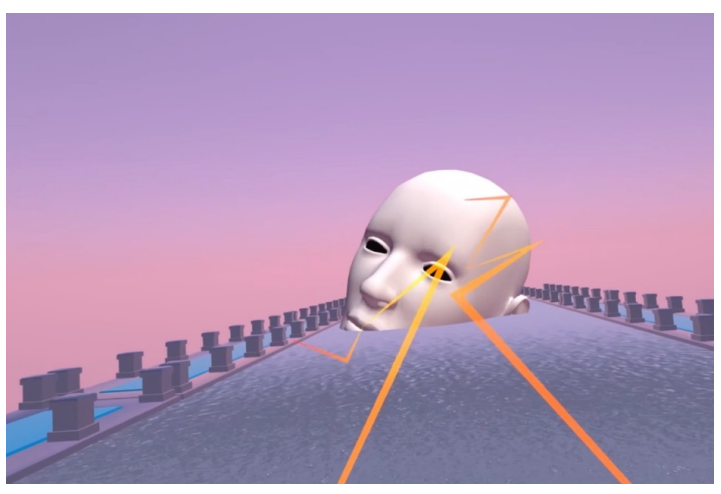

Figure 2: Zig-Zag Toy emits jagged orange beams and pulsing sounds.

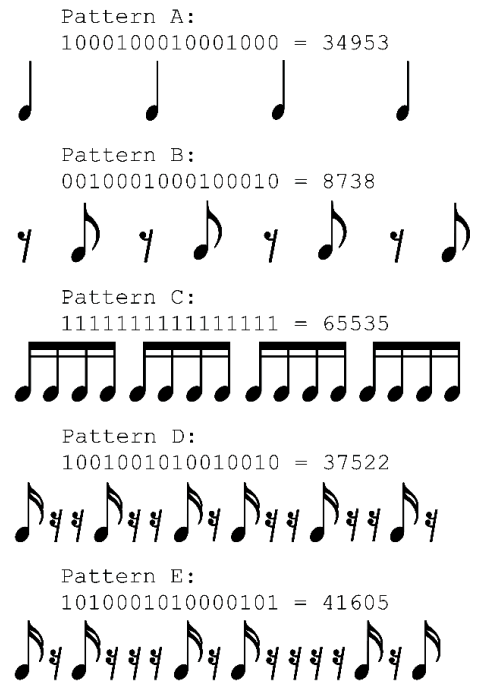

Figure 3: Zig-Zag Toy uses rhythmic patterns stored as binary sequences.

\subsection{Circle Toy}

The user can cycle between different audio-visual sound toys using the A and B buttons on the Oculus Touch controllers. This allows the selection of Circle Toy, which triggers beams of light that move in a circular pattern, which project from the hands in 3D space (Figure 3). These are blue green in color, and straight rather than jagged. They are activated using the primary trigger of the controllers.

The Circle Toy emits percussive pulsing sounds made using the Roland Cloud suite, which move in a circular rotating pattern. The sounds are designed to be complementary to the electronic dance music backdrop, but they are not synchronized to the beat, allowing patterns to be generated which are more textural in form, such as one might encounter within the field of electroacoustic composition. These sounds can be located and moved around in the spatial field by pointing the beams in the direction that one wishes the sounds to emerge from. Gesturing 
with one or two instances of Circle Toy thereby allows the user to create sweeping arcs of sound, 'painting with sound' in 3D space. The 'painting' metaphor is appropriate here due to the emphasis on gestural interaction as a means to fill the environment with additional sounds and images.

As with Zig-Zag Toy, Circle Toy also incorporates a rippling wave effect, which is generated using oscillating changes to the width of the beam along its length. The speed of circular rotation and the sound of the beam can be manipulated using the joysticks of the Oculus Touch controllers, which applies filters and pitch shift DSP effects. These transformations are accompanied by changes to the color of the beam, with oscillating visual effects being applied in a similar manner to Zig-Zag Toy, but with a different color pallet. In this way, the design of Circle Toy provides specific links between sound and image, thereby exhibiting what we might consider a synaesthetic design, in that painting with sound means painting with images, and vice-versa.

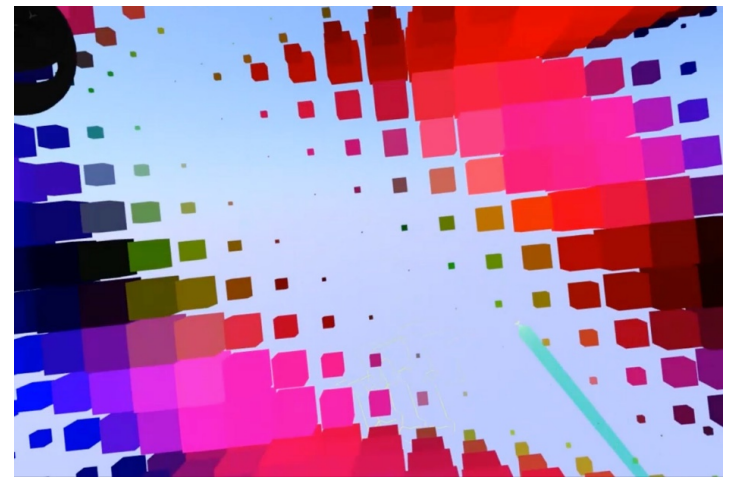

Figure 3: Circle Toy emits beams that move in a circular motion and asynchronous pulsing sounds.

\subsection{Streamer Toy}

The third audio-visual sound toy is Streamer Toy. This toy emits yellow lightning patterns accompanied by atonal droning sounds (Figure 4). The sounds were also made using the Roland Cloud to sculpt synthesizer sounds with slow attack and decay properties, thereby providing material that is suitable for creating drones, such as one might find in ambient sections of breakbeat hardcore music. Activating this sound toy with the primary trigger produces long yellow arcs that extend from the hands and fall away with gravity. The contours of these beams are linked to the hand tracking of the controllers, so that as the arcs fall away from the user, gestural movements of the hands continue to affect the position of the arc. The tips of the arcs of lightning produced by Streamer Toy emit atonal droning sounds, which are heard from these locations in terms of 3D audio. The sounds include a randomized pitch element, which provides atonal dissonance. As with the other two audio-visual sound toys discussed, the sounds can be modified using the joystick, which applies an oscillating color effect to the lightning beams.

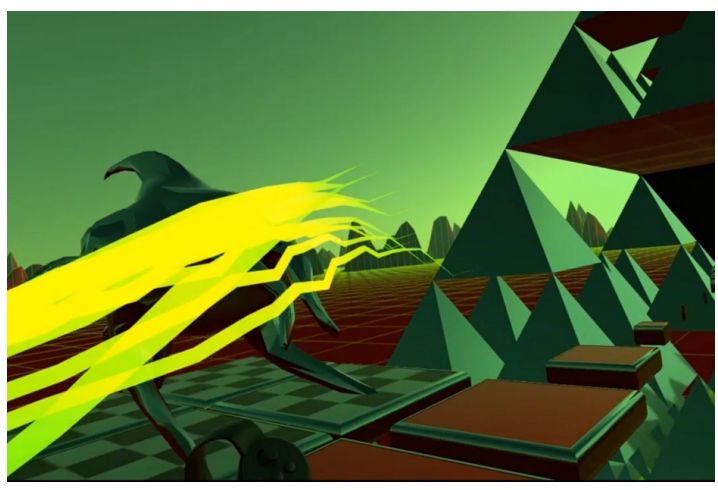

Figure 4: Streamer Toy emits yellow lightning accompanied by atonal droning sounds.

\section{Summary}

In summary, Cyberdream advances the field of VR music visualizations by demonstrating a novel set of approaches. Theoretically, the project uses a symbolic approach to music visualization, which uses the visual language associated with a music genre, in this case breakbeat hardcore, as a basis for design. This has been used to construct a journey through audio-visual environments based on the visual language of 1990s rave music. This approach could easily be used to generate many other VR music experiences, simply by examining the visual languages of other music genres and using these as a basis for design. Here, I have shown how these experiences can also interface with audiovisual sound toys and documented three possible design approaches. Next steps for this research may involve the development of further audio-visual sound toys, carrying out studies to investigate the user experience in further detail, and trialing a multiplayer version in which several participants can perform together at once. Through further research, it may be possible to realize new forms of creative audio-visual experiences using immersive technologies.

\section{REFERENCES}

[1] C.L. Berlin. 2018. Rave Art: Flyers, Invitations, and Membership Cards from the Birth of Acid House Clubs and Raves. London: Carlton Books.

[2] R.E. Cytowic. 1989. Synesthesia: A Union of the Senses. New York: SpringerVerlag.

[3] M. Faulkner (D-Fuse). 2006. Vf: Audio-Visual Art and Vj Culture. Laurence King, London.

[4] R. Fischman, 2011. Back to the Parlour, Sonic Ideas/Ideas Sónicas, 3(2): 53-66.

[5] Masters of Hardcore. 2018. Angerfist Live @ Masters of Hardcore 2018 Tournament of Tyrants. https://youtu.be/U5J92ebJfMk

[6] S. Serafin, C. Erkut, J. Kojs, N.C. Nilsson, R. Nordahl, R. 2016. Virtual Reality Musical Instruments: State of the art, design principles, and future directions, Computer Music Fournal, 40(3): 22-40.

[7] L. Turchet, R. Hamilton, A. Çamci. 2021. Music in Extended Realities, IEEE Access, 9. DOI: 10.1109/ACCESS.2021.3052931

[8] J. Weinel. 2019. Virtual Hallucinations: Projects in VJing, virtual reality and cyberculture, EVA London 2019 (Electronic Visualisation and the Arts). DOI: 10.14236/ewic/EVA2019.57

[9] J. Weinel. 2019. Cyberdream VR: Visualizing Rave Music and Vaporwave in Virtual Reality, ACM Audio Mostly 2019, University of Nottingham, UK. DOI: $10.1145 / 3356590.3356637$

[10] J. Weinel. 2020. Visualising Rave Music in Virtual Reality: Symbolic and Interactive Approaches, EVA London 2020 (Electronic Visualisation and the Arts). DOI: $10.14236 /$ ewic/EVA2020.13 